Konrad-Zuse-Zentrum

für Informationstechnik Berlin

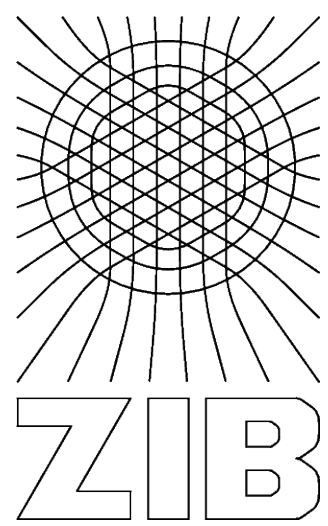

Takustraße 7

\title{
Optimization and Identification in Regional Hyperthermia
}




\title{
Optimization and Identification in Regional Hyperthermia *
}

\author{
Martin Weiser
}

October 13, 2008

\begin{abstract}
Regional hyperthermia is a cancer therapy aiming at heating tumors using phased array applicators. This article provides an overview over current mathematical challenges of delivering individually optimal treatments. The focus is on therapy planning and identification of technical as well as physiological quantities from MR thermometry measurements.
\end{abstract}

AMS MSC 2000: 92C50,65M32

Keywords: hyperthermia treatment planning, identification, MR thermometry, interior point methods

\section{Introduction}

Hyperthermia is a cancer therapy aiming at heating tumors in order to either destroy cancer cells directly or to make them more susceptible to an accompanying radio- or chemotherapy. Several different mechanisms are responsible for the cytotoxic effect of hyperthermia $[7,9]$. Different technological means are used to deliver heat to the tumor. Heating techniques applied mostly to smaller or more superficial tumors are RF ablation [1], highly focused ultrasound [11], or magnetic nanoparticles [10].

*Supported by the DFG Research Center Matheon "Mathematics for key technologies" in Berlin. 


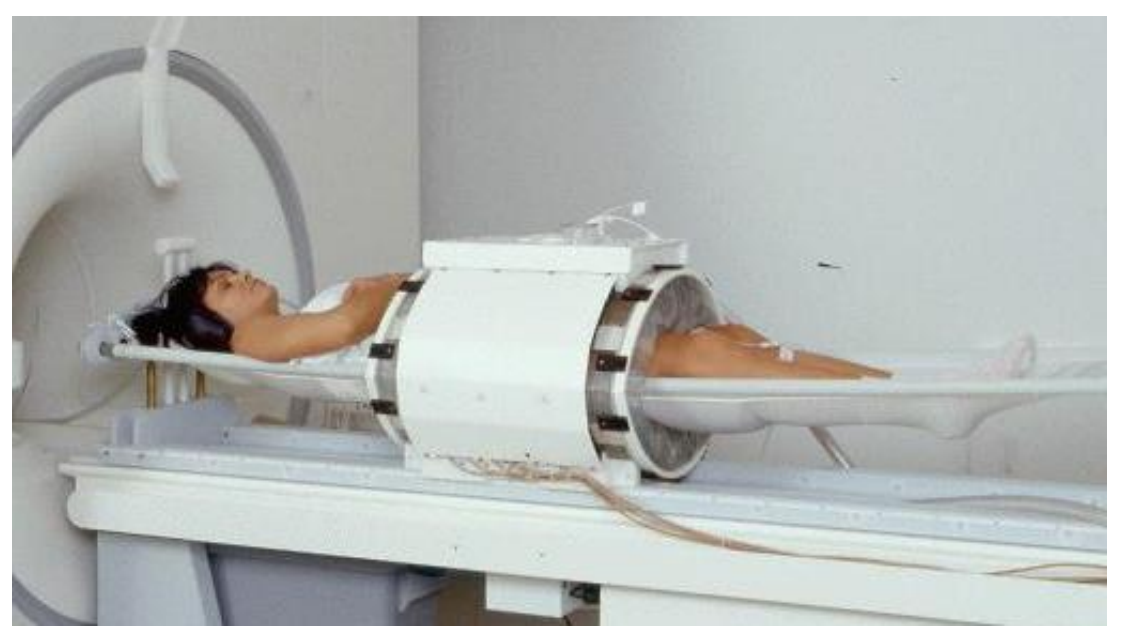

Figure 1: Hybrid MR-hyperthermia treatment system in Berlin (Charité).

For large, deeply seated, and inoperable tumors, regional hyperthermia is used [25]. Here, a time-harmonic electrical interference field is generated by a phased array of antennas which can be controlled individually by amplitude and phase. During a treatment session of up to two hours, the patients's tissue absorbs energy from the electrical field. The heat is distributed inside the body, resulting in a leveraged temperature distribution. By now, several clinical studies have demonstrated the beneficial effect of regional hyperthermia.

In order to deliver an optimal therapy, the phased array applicator needs to be controlled in such a way that the tumor is maximally heated without damaging healthy tissue by excessive temperatures. Due to individually varying tumor location and patient geometry, individual therapy planning is necessary. The physical processes of field interference and heat distribution inside the very heterogeneous human body is too complex to be optimized manually. Thus, optimization algorithms are required for therapy planning, which is considered in Section 2.

The reliability of the mathematical optimization depends on the accuracy of the models describing the physical situation. As is quite common in biomedical settings, in particular the physiological parameters are individually varying to a significant amount, such that a priori models are subject to significant modeling errors. Online parameter identification based on magnetic resonance imaging (MRI) may be a remedy. This is considered in Section 3.

Due to both, the technical complexity of the HF power generation and distribution and the irregular geometry, modeling errors for computing the electrical field are virtually unavoidable. Thus, even accurate solution of Maxwell's 
equations will not provide the actual electrical field. Again, MRI can be used for identification of the applicator parameters, which is considered in Section 4.

\section{Therapy Planning with Interior Point Meth- ods}

The distribution of heat in the patient's body is a dynamic process. However, the transient heating phase takes about 15 minutes, while the duration of a single treatment session is about two hours. For this reason, usually only the steady state of the temperature distribution is optimized, which results in a significantly simpler optimization task.

Objective. The ultimate goal of hyperthermia is the destruction of the tumor. The thermal damage inflicted on cancer cells follows the Arrhenius law [3], with a rate constant

$$
k(T)=A e^{-\frac{\Delta E}{R T}} .
$$

Frequency factor $A$ and activation energy $\Delta E$ depend on the tissue type, and $R$ is the universal gas constant. Now an estimate $s$ of the fraction of surviving cells is given by $\dot{s}(t, T)=-k(T) s(t, T)$ with $s(0, T)=1$ and hence $s(\tau, T)=\exp (-k(T) \tau)$. The cost functional to be minimized is then the total number of surviving cancer cells:

$$
\min \int_{\text {tumor }} \exp \left(A \tau e^{-\frac{\Delta E}{R T}}\right) d x
$$

The thermal isoeffect dose is an established quantity for assessing the therapeutic benefit of a treatment $[3,18]$. Treatment planning based on the tumor cell survival has been proposed for thermoseed placement [21], but up to now rather ad hoc cost functionals based on the temperature distribution $[1,15]$ or on the absorption rate density [16] have been used for regional hyperthermia.

Constraints. The state equation is given by the bio-heat transfer equation (BHTE) describing the distribution of heat in the human body. The most simple variant dates back to [17]:

$$
\begin{gathered}
\operatorname{div}(\kappa \nabla T)+c_{b} w\left(T_{a}-T\right)+\frac{\sigma}{2}|E|^{2}=0 \quad \text { in } \Omega \\
\kappa \partial_{n} T=h\left(T_{\text {out }}-T\right) \quad \text { on } \partial \Omega
\end{gathered}
$$




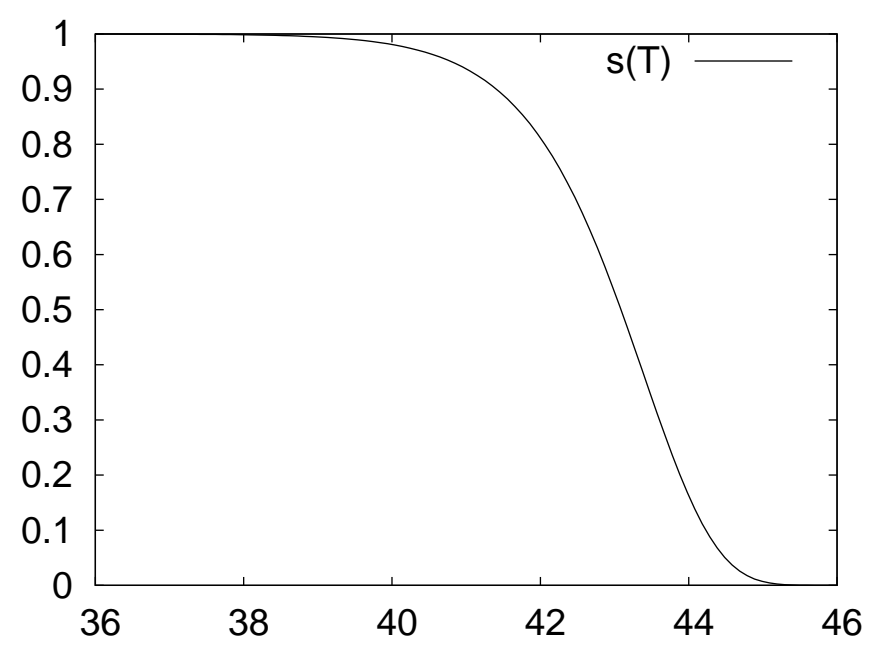

Figure 2: Arrhenius model of fraction of surviving cancer cells versus temperature.

Here, $\kappa$ is the tissue's heat conductivity, $c_{b}$ heat capacity of blood, $w$ the perfusion, and $\sigma$ the electric conductivity. The domain $\Omega$ covers the part of the patient's body that is relevant for therapy planning.

The electrical field $E$ satisfies the time-harmonic Maxwell's equation

$$
\operatorname{curl} \frac{1}{\mu} \operatorname{curl} E-\omega^{2} E=i \omega \sum_{k=1}^{n} u_{k} J_{k} \quad \text { in } \Omega_{E},
$$

where the $n$ antenna currents $u_{k} J_{k}$ are determined by the applicator control $u \in \mathbb{C}^{n}$. The computational domain $\Omega_{E}$ for (4) includes $\Omega$ as well as the water bolus, the applicator antennas, and a region of air around the applicator. On the boundary $\partial \Omega_{E}$, approximately transparent boundary conditions are prescribed to avoid artificial reflections.

Since (4) is linear and its solution is expensive, inside the optimization algorithm the electric field $E=V u$ is computed as a superposition of antenna profiles $V_{k}$ satisfying (4) with $u_{i}=\delta_{i k}$. Thus, equation (4) needs to be solved only $n$ times.

Of course, healthy tissue should not be damaged by too high temperatures. Therefore, an upper bound is imposed on the temperature:

$$
T \leq T_{\lim }
$$

Typical values for $T_{\lim }$ are $44^{\circ} \mathrm{C}$ for muscle, fat, and bone tissues, and $42^{\circ} \mathrm{C}$ for more sensitive organs such as bladder or intestine. 
Material properties. The wavelength of the electrical field in the body is around $30 \mathrm{~cm}$, such that the absorption rate density cannot be focused narrowly on the tumor alone. In fact, the most important effect of treatment planning is to construct negative interference in regions where otherwise hot spots would limit the overall power that can be deposited in the patient. Nevertheless, temperatures above $45^{\circ} \mathrm{C}$ are rarely achieved. In contrast to $\mathrm{RF}$ ablation and focused ultrasound therapies, electrical and thermal tissue properties do not change significantly in this low temperature range. For this reasons, these values are simply taken as constants depending only on tissue type as given in $[2,15]$.

On the other hand, the perfusion $w$ depends on the temperature due to autoregulation capabilities of the tissue. Moreover, at least in abdominal hyperthermia, the systemic thermoresponse seems to play a significant role. Different perfusion models have been proposed, covering a broad spectrum of homogenized and discrete vascular models $[8,12,15,23]$.

Function space interior point methods. Combining (1)-(5) we end up with a nonlinear stationary state-constrained scalar PDE optimization problem. Due to the distributed state constraint (5), the Lagrange multipliers are usually only measure-valued, which makes the theoretic and algorithmic treatment intricate. Following [24], we apply primal interior point methods directly to the infinite dimensional optimization problem. The inequality constraints (5) are moved into the objective using an integrated barrier function $b$ :

$$
\begin{array}{rlrl}
\min \int_{\text {tumor }} \exp \left(A \tau e^{-\frac{\Delta E}{R T}}\right) d x+\mu \int_{\Omega} b\left(T_{\lim }-T\right) d x & & \\
\text { s.t. } \operatorname{div}(\kappa \nabla T)+c_{b} w\left(T_{a}-T\right)+\frac{\sigma}{2}|E(u)|^{2} & =0 & & \text { in } \Omega \\
\kappa \partial_{n} T & =h\left(T_{\text {out }}-T\right) & & \text { on } \partial \Omega
\end{array}
$$

This translates the state-constrained problem into a parametric unconstrained problem, the solutions of which form the so-called central path. Existence and convergence of the central path to the solution of the original problem have been shown in [20] for rational barrier functions of sufficiently large order. An efficient damping strategy has been worked out in [19].

The central path is followed by a numerical continuation method employing an inexact Newton corrector. Note that both pathfollowing and Newton corrector are working on the continuous problem setting, using adaptive finite element discretization only on the lower level for solving linear KKT operator 


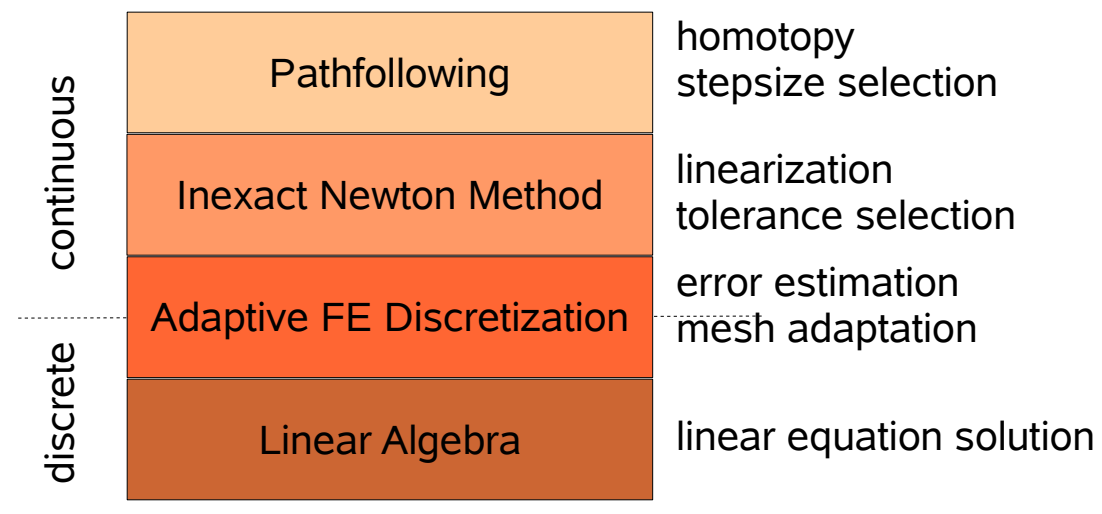

Figure 3: Structure of the function space interior point method.

equation systems, see Fig 3. The linear PDE systems are solved to an accuracy that is chosen by the inexact Newton corrector such that linear convergence towards the central path is obtained. An example of optimized temperature distributions is shown in Fig. 4.

\section{Perfusion Identification}

One of the most important but least known quantities in regional hyperthermia therapy planning is the perfusion $w$. It varies highly between individuals and depends on the patient's condition at treatment time as much as on the temperature distribution. For this reason, a priori perfusion models used for therapy planning are bound to incur significant quantitative errors. A possible remedy is to measure the perfusion during the treatment and to adapt the applicator control based on a reoptimization with updated model in a closed-loop fashion. However, 3D distributed direct perfusion measurements require the injection of contrast agents, which is possible at most one or two times during a therapy seesion.

In contrast, MR based thermometry measurements [6] are readily available online in a hybrid MR-hyperthermia system (see Fig.1). The 3D voxel data $m$ can be obtained approximately every other minute from proton resonance frequency shifts. Since $m \approx a T+b w$ is affected by both, temperature $T$ and perfusion $w$, we can formulate the regularized identification problem

$$
\min \frac{1}{2}\|a T+b w-m\|_{L^{2}(\Omega)}^{2}+\frac{\alpha}{2}\left\|w-w_{\text {ref }}\right\|^{2}
$$




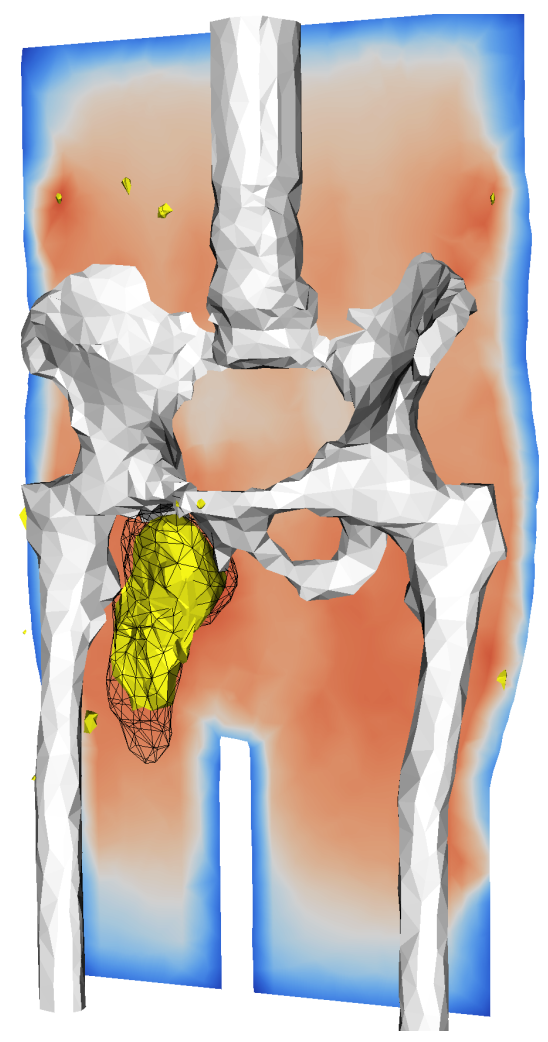

Figure 4: Optimized emperature distribution inside a patient's body. The tumor is drawn with black lines. Isothermal surfaces are shown for $43.5^{\circ} \mathrm{C}$. Note that the hot spots in healthy tissue limit the total power and hence the tumor temperature.

subject to the bio heat transfer equation

$$
\operatorname{div} \kappa \nabla T+c_{b} w\left(T_{a}-T\right)+\frac{\sigma}{2}|E|^{2}=0
$$

The coefficients $a$ and $b$ are assumed to be equal in muscle tissue whereas $a=0$ holds for fatty tissue. This PDE-constrained optimization problem has been considered in [4]. Preliminary numerical experiments on a 2D cross section of a clinical patient showed promising results. In particular, the temperature deviation between simulation and a temperature probe positioned in the vicinity of the tumor was decreased by a factor of three, and the low-perfused necrotic core of the tumor that had not been modeled in the reference perfusion was identified.

Taking a closer look at the structure of the identification problem, however, reveals a possible non-uniqueness of the solution due to the nonlinearity of the 
bio heat transfer equation. Neglecting heat diffusion and regularization, (6) and (7) reduce to

$$
\min \frac{1}{2}\left(a\left(\frac{\sigma}{2 c_{b} w}|E|^{2}+T_{a}\right)+b w-m\right)^{2}
$$

in each point in space. In fatty tissue with $a=0, w=m / b$ is the only physiological solution. But in muscle tissue with $a=b$, and in case

$$
T_{a}-\frac{m}{a}>\sqrt{\frac{2 \sigma}{c_{b}}}|E|,
$$

two separate local minima of (8) exist. Since diffusion is comparatively small and moderate regularization tends to only shift the local minima by some amount, two different temperature and perfusion values, respectively, may be expected to satisfy the BHTE and to match the measurement simultaneously. This is actually observed in numerical examples.
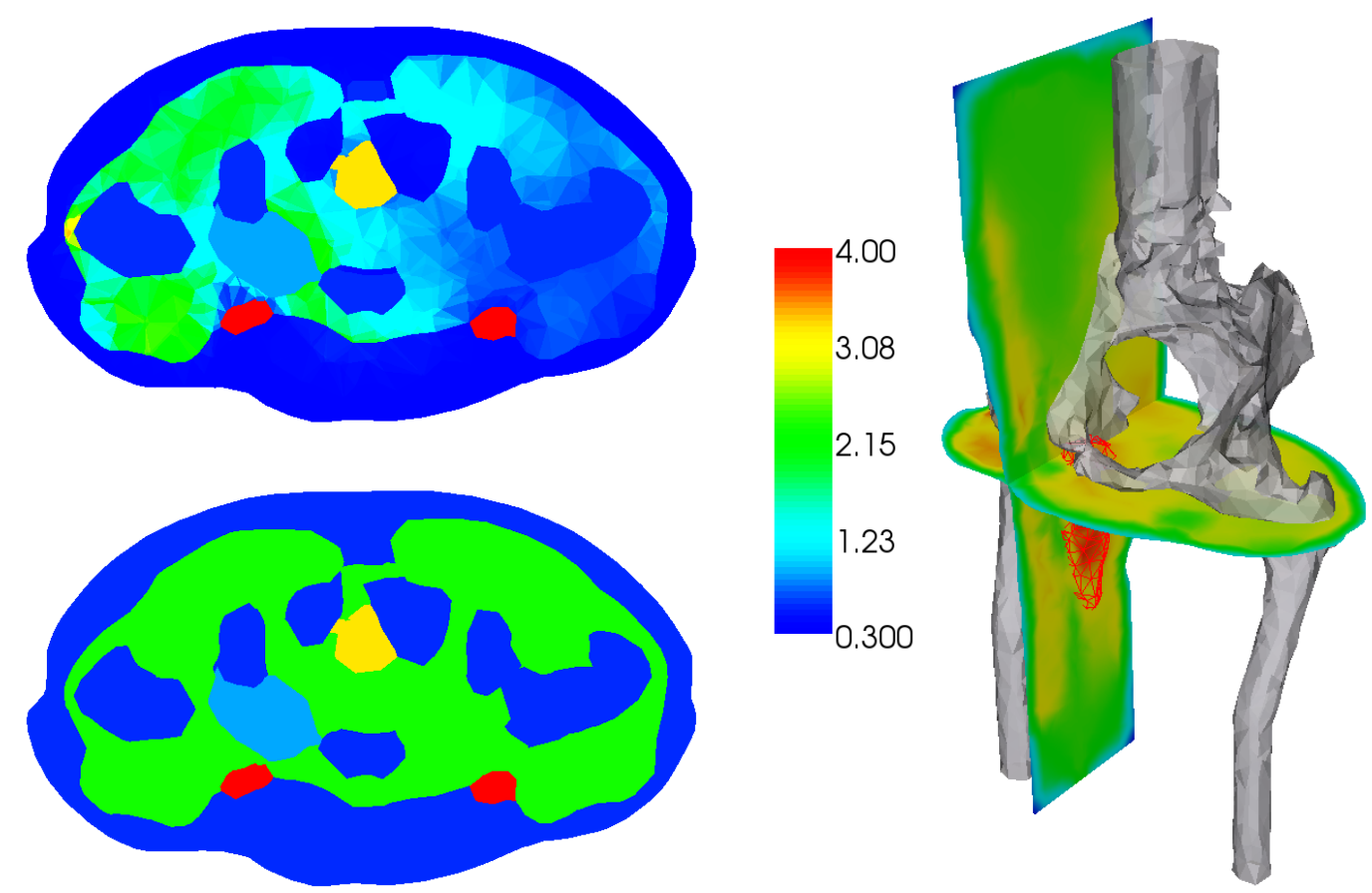

Figure 5: Example setting for misleading perfusion identification. Top left: actual perfusion used to compute $m$. Bottom left: reference perfusion. Right: 3D geometry with simulated temperature distribution. Cross-cuts shown on the left are taken from the horizontal plane also shown on the right. Muscle tissue appears green. 
Fig. 5 shows one situation where the identification seems to be trapped in the wrong local minimum of (8). Simulated measurement data has been computed from the actual perfusion (top left), the associated temperature given by the solution of the BHTE, and relative noise. The reference perfusion used for moderate $L^{2}$ regularization is constant on each tissue type. Identification results are given in Fig. 6. Perfusion and temperature values are in reasonable agreement with the actual values on the left of the cross section. However, in the central area and on the right, perfusion is overestimated and temperature is underestimated in muscle tissue. Note that this is not due to strong regularization, as the same effect is visible in the low noise situation with small regularization factor $\alpha$. In fact, on the right hand side, the structure of the actual perfusion is still present in the low noise identified perfusion, but with too large values.

Computing the posterior probability distribution of $w$ and $T$ would give a more complete picture and reveal the non-uniqueness. However, the clinical benefit of such an approach is questionable, since therapeutical decisions have to be made based on temperature and perfusion values. It seems as if the acquisition of complementary measurement data for disambiguation would be more useful.

\section{Antenna Profile Adaptation}

A second significant source of therapy planning errors is the behavior of the hyperthermia system itself. First of all, the fidelity of the HF power generator output is less than optimal. Second, the feed network consisting of coax cables contains plug-in connectors with unknown and varying contact resistances, at which the HF signal is partially reflected. Finally, the electrical field itself is affected by impurities in the water bolus changing the water's conductivity, by simplifications of the applicator geometry in the FE model, and by any mobile conducting material in the vicinity of the applicator. For this reason, the electrical fields emanating from each antenna individually (antenna profiles) are computed to moderate accuracy at best [5], leading to significant errors in the computed energy absorption as shown in Fig. 7.

A quite radical approach is to drop the solution of Maxwell's equation altogether and instead try to identifiy the action matrix $M=V^{H} V$ from MR thermometry measurements, as has been proposed in [13,14]. This can be done in each voxel. However, a quick calculation shows that for an applicator with $n$ channels the Hermitian matrix $M$ contains $n^{2}$ degrees of freedom. A full 


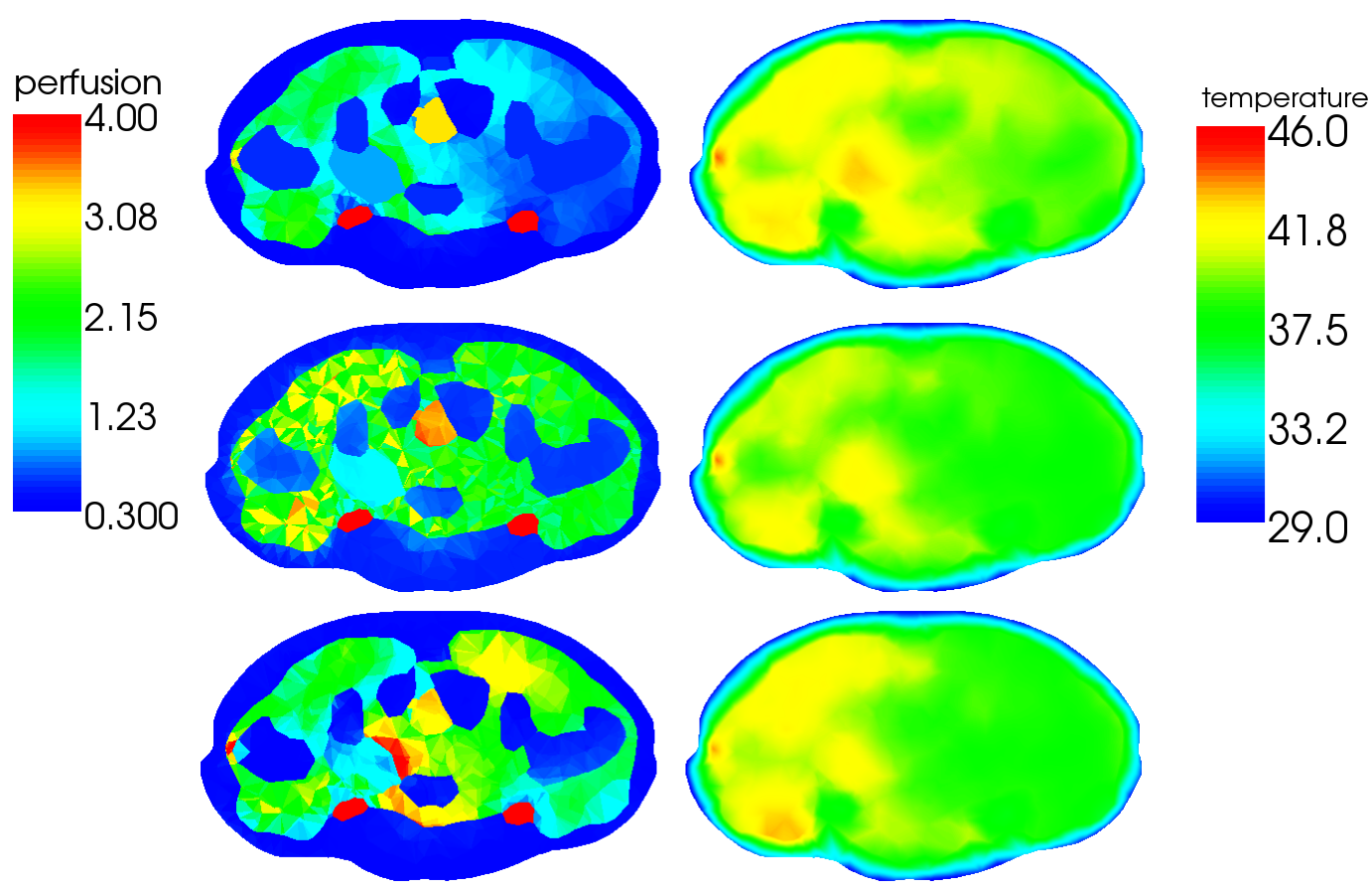

Figure 6: Identification results. Left: perfusion. Right: temperature. Top row: actual values used to compute the measurement $m$. Middle row: identified values for high noise data with strong regularization. Bottom row: identified values for low noise data with small regularization.

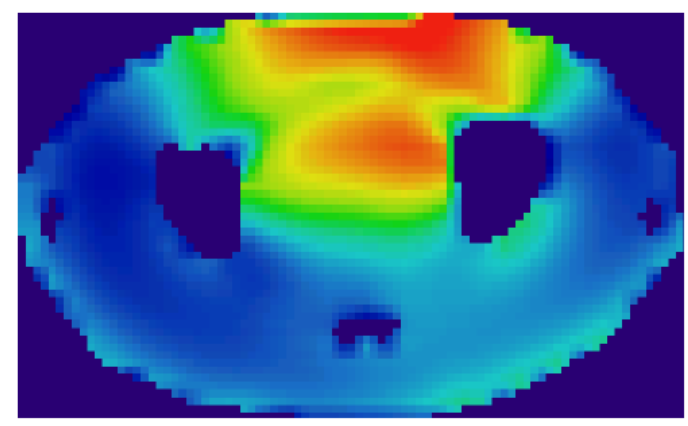

(a)

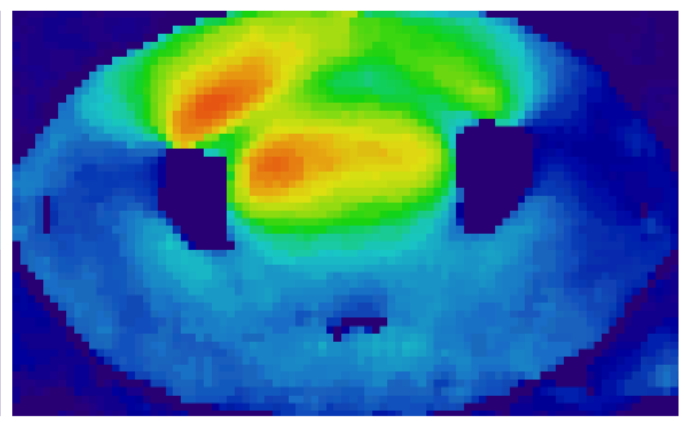

(b)

0

8.3

16.7

25.1

$33.5[\mathrm{~W} / \mathrm{kg}]$

Figure 7: Absorption rate density in a cross section of a heterogeneous phantom. Left: predicted ARD from FDTD computations of the antenna profiles. Right: ARD computed from MR thermography measurements. 
identification would require at least $n^{2}$ different temperature measurements spanning the whole control space. Modern applicators employed in clinical practice feature 12 channels, such that, assuming one minute per MR measurement, a patient would have to endure two hours of heating with clinically questionable absorption rate patterns before the model is adjusted.

Therefore, a less radical approach has been proposed in [22], where MR thermometry measurements are used only to adapt a priori computed antenna profiles to the measurements. Moreover, instead of the action matrix $M=V^{H} V$, the antenna profile matrix $V \in \mathbb{C}^{3 \times n}$ itself is adapted. Note that $V$ contains only $6 n$ real degrees of freedom, of which three have no thermal impact (simultaneous phase shifts and simultaneous rotation of field vectors). For larger numbers of channels, the reduction in model size should be significant, but leads to a nonlinear identification problem.

From the instationary BHTE we obtain

$$
c \frac{T(t+\delta t)-T(t)}{\delta t} \approx c \dot{T}=\operatorname{div}(\kappa \nabla T)+c_{b} w\left(T_{a}-T\right)+\frac{\sigma}{2}|E|^{2} .
$$

In the most simple approach, diffusion and perfusion are neglected, such that approximate ARD measurements are available from two successive MR thermometry measurements as

$$
\mathrm{ARD}^{m}=c \frac{T(t+\delta t)-T(t)}{\delta t} .
$$

Defining the data mismatch for a certain applicator control $u$ as

$$
F\left(V, u, \mathrm{ARD}^{\mathrm{m}}\right)=u^{H} V^{H} V u-\frac{\sigma}{2} \mathrm{ARD}^{m}
$$

we can formulate a nonlinear least squares problem

$$
\min \sum_{i=1}^{k} F\left(V, u_{i}, \mathrm{ARD}_{i}^{m}\right)^{2}
$$

for $k$ different controls with associated measurements. Note that due to the low number of available measurements compared to the degrees of freedom in $V,(9)$ is highly underdetermined. Therefore, instead of (9), the nonlinear total least squares problem

$$
\min _{V}\left\|V-V_{0}\right\|_{F}^{2} \quad \text { subject to } \sum_{i=1}^{k} F\left(V, u_{i}, \mathrm{ARD}_{i}^{m}\right)^{2}=\min
$$



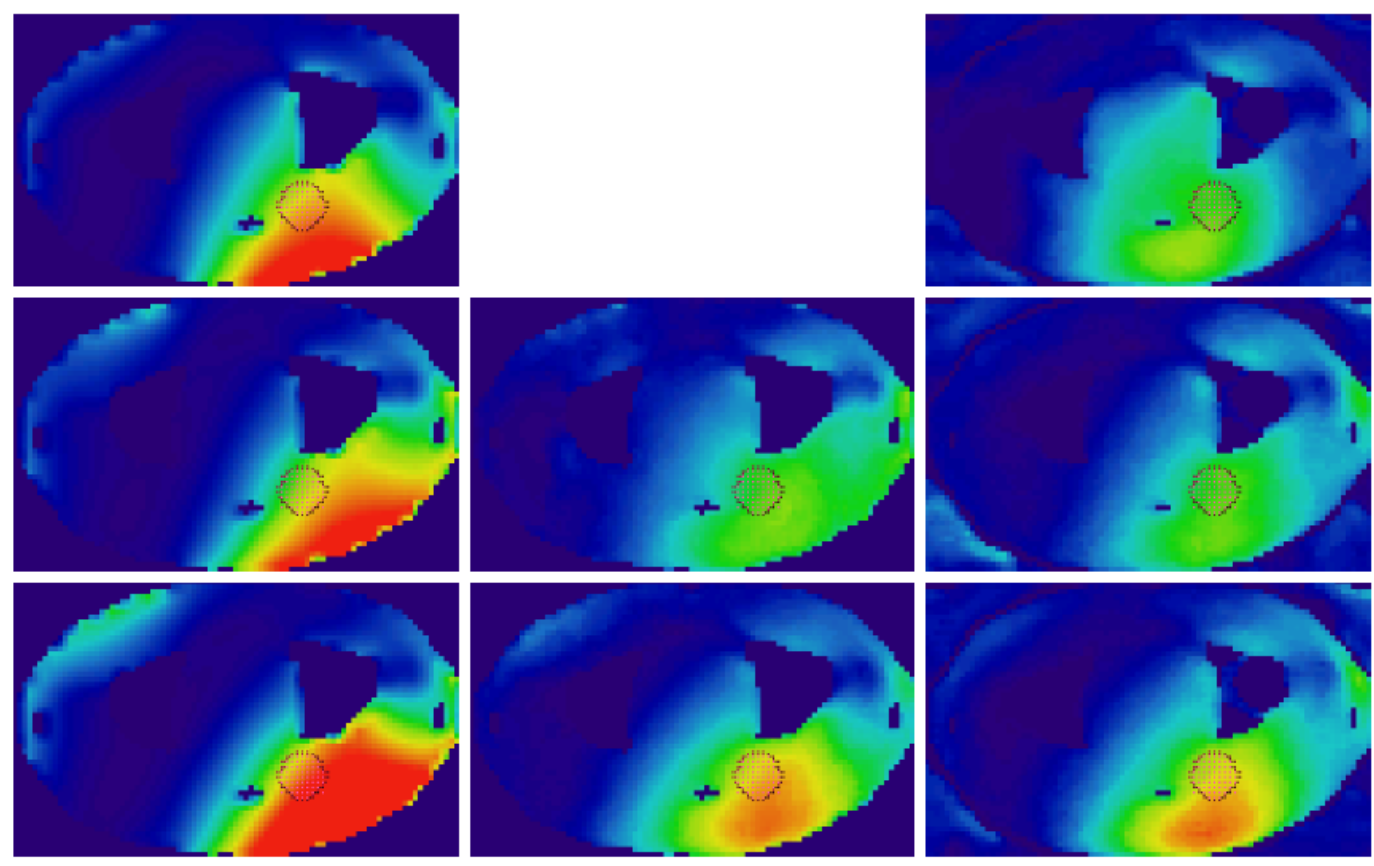

Figure 8: Closed loop results. Left column: ARD prediction based on solution of Maxwell's equation (4). Middle column: ARD prediction for next control based on adapted antenna profiles. Right column: ARD measurements for current control. Controls for each row successively have been selected by maximizing ARD in the dotted target region based on the adapted antenna profiles.

is solved approximately by a Gauß-Newton method. A closed loop controller is then given by the following scheme:

compute $V_{0}$ by solving (4)

for $k=1, \ldots$

compute $u_{k}$ by minimizing (1) based on $V_{k-1}$

apply $u_{k}$ and measure $\mathrm{ARD}_{k}^{m}$

solve (10) for $V_{k}$

The first few steps of this loop applied to heating a heterogeneous phantom are shown in Fig. 8. The reduction of the discrepancy between prediction and measurement is clearly visible.

Phantom experiments in [22] demonstrated a reduction of ARD prediction error by a factor of two and more, but showed no significant difference between adapting either $M$ or $V$. More importantly, devising new applicator controls based on adapted antenna profiles during actual therapy recently led to a significant increase of tumor temperature, see Fig.9. 

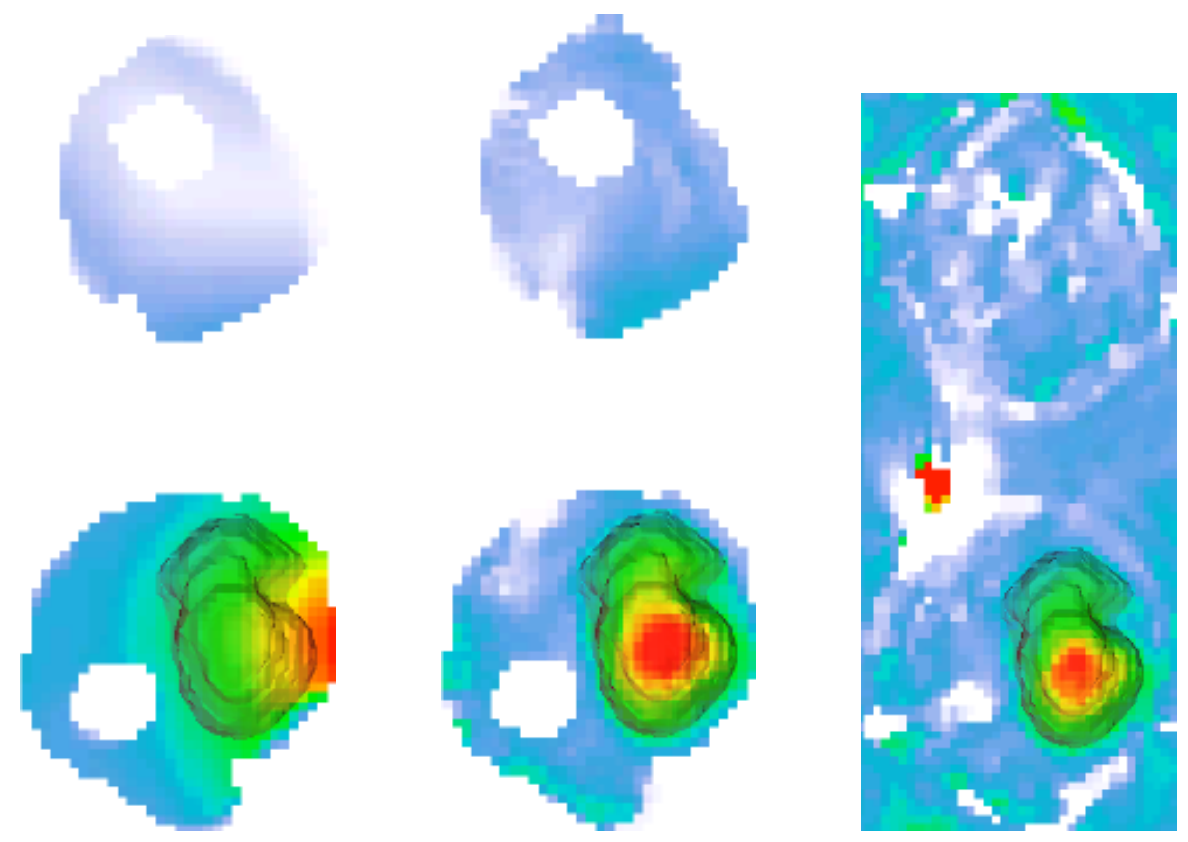

Figure 9: Improved tumor heating due to online reoptimization based on adapted antenna profiles. Cross sections of a patient with color coding by ARD, tumor region shaded. Left: applicator control $a$ optimized with antenna profiles from FDTD, computed with adapted antenna profiles. Center: applicator control $b$ optimized with adapted antenna profiles. Right: MR measurement for the applicator control $b$.

\section{Conclusion}

Therapy planning for regional hyperthermia still poses interesting problems to modelling and numerical analysis. From a practical point of view, available algorithms are possible to perform treatment design reasonable well. Despite significant uncertainties in the models, the temperature prediction for regional hyperthermia in the pelvic region is surprisingly accurate with deviations of around $0.5^{\circ} \mathrm{C}$ to $1^{\circ} \mathrm{C}$ from temperature probe values. Nevertheless, since the therapeutic effect can crucially depend even on such small temperature differences, improving the model and prediction accuracy by parameter identification is very important. This is particularly the case in more complex situations such as abdominal hyperthermia or special vascular structures where interregional and systemic thermoregulation mechanisms play an important role.

While the identification or at least adaptation of electrical fields from MR 
thermography measurements is quite promising, identification of perfusion seems to require different or additional measurement data to disambiguate the results.

Acknowledgement. The author gratefully acknowledges longstanding collaboration with P. Wust, J. Gellermann, and M. Weihrauch from Charité and with P. Deuflhard and A. Schiela from ZIB.

\section{References}

[1] I. Altrogge, T. Preusser, T. Kröger, C. Büskens, P. Pereira, D. Schmidt, and H.-O. Peitgen. Multiscale optimization of the probe placement for radiofrequency ablation. Acad. Radiol., 14(11):1310-1324, 2007.

[2] P. Deuflhard, M. Weiser, and M. Seebaß. A new nonlinear elliptic multilevel FEM applied to regional hyperthermia. Comput. Vis. Sci., 3(3):115$120,2000$.

[3] W.C. Dewey. Arrhenius relationships from the molecule and cell to the clinic. Int. J. Hyperthermia, 10(4):457-483, 1994.

[4] T. Gänzler, S. Volkwein, and M. Weiser. SQP methods for parameter identification problem arising in hyperthermia. Optim. Meth. Softw., 21(6):869-887, 2006.

[5] J. Gellermann, M. Weihrauch, C.H. Cho, W. Wlodarczyk, H. Fähling, R. Felix, V. Budach, M. Weiser, J. Nadobny, and P. Wust. Comparison of MR-thermography and planning calculations in phantoms. Medical Physics, 33:3912-3920, 2006.

[6] J. Gellermann, W. Wlodarczyk, A. Feussner, H. F"ahling, J. Nadobny, B. Hildebrandt, R. Felix, and P. Wust. Methods and potentials of magnetic resonance imaging for monitoring radiofrequency hyperthermia in a hybrid system. Int. J. Hyperthermia, 21(6):497-513, 2005.

[7] B. Hildebrandt, P. Wust, O. Ahlers, A. Dieing, G. Sreenivasa, T. Kerner, R. Felix, and H. Riessa. The cellular and molecular basis of hyperthermia. Crit. Rev. Oncol. Hematol., 43:33-56, 2002.

[8] R. Hochmuth and P. Deuflhard. Multiscale analysis for the bio-heattransfer equation - the nonisolated case. Mathematical Models and Methods in Applied Sciences, 14(11):1621-1634, 2004. 
[9] M.R. Horsman. Tissue physiology and the response to heat. Int. J. Hyperthermia, 22:197-203, 2006.

[10] A. Jordan, R. Scholz, K. Maier-Hauff, M. Johannsen, P. Wust, J. Nadobny, H. Schirra, H. Schmidt, S. Deger, S. Loening, W. Lanksch, and R. Felix. Presentation of a new magnetic field therapy system for the treatment of human solid tumors with magnetic fluid hyperthermia. J. Magnetism Magn. Mater., 225:118-126, 2001.

[11] J.E. Kennedy, G.R. ter Haar, and D. Cranston. High intensity focused ultrasound: surgery of the future? British Journal of Radiology, 76:590599, 2003.

[12] A. Kotte. Design of a numerical model for describing the heat transfer due to vascular trees. PhD thesis, University Utrecht, 1998.

[13] M.E. Kowalski, B. Behnia, A.G. Webb, and J.-M. Jin. Optimization of electromagentic phased-array hyperthermia via magnetic resonance temperature estimation. IEEE Trans. Biomed. Eng., 49:1229--1241, 2002.

[14] M.E. Kowalski and J.-M. Jin. A temperature-based feedback control system for electromagnetic phased-array hyperthermia: theory and simulation. Phys. Med. Biol., 48:633-651, 2003.

[15] J. Lang, B. Erdmann, and M. Seebaß. Impact of Nonlinear Heat Transfer on Temperature Control in Regional Hyperthermia. IEEE Trans. Biomed. Eng., 46(9):1129-1138, 1999.

[16] P. Maass and R. Ramlau. Wavelet-accelerated regularization methods for hyperthermia treatment planning. Int. J. Imaging Systems Tech., 7:191-199, 1996.

[17] H.H. Pennes. Analysis of tissue and arterial blood temperatures in the resting human forearm. J. Appl. Phys., 1:93-122, 1948.

[18] G.L. Rosner, S.T. Clegg, D.M. Prescott, and M.W. Dewhirst. Estimation of cell survival in tumours heated to nonuniform temperature distributions. Int. J. Hyperthermia, 12(2):223-239, 1996.

[19] A. Schiela. An interior point method in function space for the efficient solution of state constrained optimal control problems. ZIB Report 07-44, 2007.

[20] A. Schiela. An extended mathematical framework for barrier methods in function space. Report 08-08, ZIB, 2008. 
[21] D.T. Tompkins, R. Vanderby, S.A. Klein, W.A. Beckman, R.A. Steeves, and B.R. Paliwal. The use of generalized cell-survival data in a physiologically based objective function for hyperthermia treatment planning: a sensitivity study with a simple tissue model implanted with an array of ferromagnetic thermoseeds. Int. J. Radiation Oncology Biol. Phys., 30(4):929-943, 1994.

[22] M. Weihrauch, P. Wust, M. Weiser, J. Nadobny, S. Eisenhardt, V. Budach, and J. Gellermann. Adaptation of antenna profiles for control of MR guided hyperthermia (HT) in a hybrid MR-HT system. Med. Phys., 34(12):4717-4725, 2007.

[23] S. Weinbaum and L.M. Jiji. A new simplified bioheat equation for the effect of blood flow on local average tissue temperature. ASME J. Biomech. Eng., 107:131-139, 1985.

[24] M. Weiser and P. Deuflhard. Inexact central path following algorithms for optimal control problems. SIAM J. Control Optim., 46(3):792-815, 2007.

[25] P. Wust, B. Hildebrandt, G. Sreenivasa, B. Rau, J. Gellermann, H. Riess, R. Felix, and P.M. Schlag. Hyperthermia in a combined treatment of cancer. The Lancet Oncology, 3:487-497, 2002. 